H. Morikawa

Nagoya Math. J.

Vol. 33 (1968), 133-135

\title{
ON COMMUTATIVE COMPOSITIONS DETERMINED BY THEIR ORIGINS
}

\author{
HISASI MORIKAWA
}

1. Let $K$ be the universal domain. Let $G$ be a finite additive group of odd order $|G|$ and $X_{a}(a \in G)$ be indeterminates indexed by the elements in $G$. We mean by $P_{G}$ the projective space $\operatorname{Proj}_{k}\left(K\left[\left(X_{a}\right)_{a \in G}\right]\right)$. Denote by $\delta_{-1}$ and $\tau_{b}(b \in G)$ the automorphisms of $P_{G}$ of which duals $\delta_{-1}^{*}$ and $\tau_{b}^{*}$ are the ring-automorphisms of $Z\left[\left(X_{a}\right)_{G}\right]$ such that

$$
\delta_{-1}^{*}\left(X_{a}\right)=X_{-a}, \quad \tau_{b}^{*}\left(X_{a}\right)=X_{b+a} \quad(a, b \in G) .
$$

For the sake of simplicity we denote briefly

$$
x^{-1}=\delta^{-1}(x), \quad x(b)=\tau_{b}(x) \quad\left(x \in P_{G}, \quad b \in G\right) .
$$

Definition 1. 1 Let $e=\left(e_{a}\right)_{G}$ be a point on $P_{G}$ satisfying

$$
e_{-a}=e_{a} \quad(a \in G) .
$$

Then two points $x=\left(x_{a}\right)_{G}$ and $y=\left(y_{a}\right)_{G}$ are called to be composable with respect to $e$, if there exist two vectors $u=\left(u_{a}\right)_{G}$ and $v=\left(v_{a}\right)_{G}$ such that

(2)

$$
\operatorname{rank}\left(\begin{array}{c}
\left(e_{-a+b} e_{a+b}\right)_{G, G}\left(y_{-a+d} y_{a+d}\right)_{G, G} \\
{ }^{t}\left(x_{-c+b} x_{c+b}\right)_{G, G}\left(u_{-c+d} v_{c+d}\right)_{G, G}
\end{array}\right)=\operatorname{rank}\left(e_{-a+b} e_{a+b}\right)_{G, G},
$$

where $\left(e_{-a+b} e_{a+b}\right)_{G, G},\left(x_{-a+b} x_{a+b}\right)_{G, G},\left(y_{-a+b} y_{a+b}\right)_{G, G}$ and $\left(u_{-a+b} v_{a+b}\right)_{G, C}$ are $|G|+$ $|G|$-matrices of which $(a, b)$-components are $e_{-a+b} e_{a+b}, x_{-a+b} x_{a+b}, y_{-a+b} y_{a+b}$ and $u_{-a+b} v_{a+b}$, respectively, $(a, b \in G)$.

Since the order $|G|$ is odd, the pair $(-a+b, a+b)$ runs over all the elements in $G \times G$. Therefore the system of equations

$$
u_{-a+b} v_{a+b}=u_{-a+b}^{\prime} v_{a+b}^{\prime} \quad(a, b \in G)
$$

implies $u_{a} / u_{a}^{\prime}=u_{b} / u_{b}^{\prime}, v_{a} / v_{a}^{\prime}=v_{b} / v_{b}^{\prime}(a, b \in G)$. Namely the point $u=\left(u_{a}\right)_{G}$ and $v=\left(v_{a}\right)_{G}$ in (2) are uniquely determined by $x$ and $y$ as points in $P_{G}$.

Received February 27, 1968. 
Definition 1. 2. If $x=\left(x_{a}\right)_{G}$ and $y=\left(y_{a}\right)_{G}$ are composable with respect to $e$, we denote by $x \circ y$ the unique point $v=\left(v_{a}\right)_{a}$ given in (2) and call it the composition of $x$ and $y$ with respect to $e$.

Proposition 1. 3. If $x=\left(x_{a}\right)_{G}$ and $y=\left(y_{a}\right)_{G}$ are composable with respect to $e$, them it follow

$$
\text { (3) } \begin{aligned}
\operatorname{rank} & \left(\begin{array}{l}
\left(e_{-a+b} e_{a+b}\right)_{G, G}\left(y_{-a+d} y_{a+d}\right)_{G, G} \\
{ }^{t}\left(x_{-c+b} x_{c+b}\right)_{G, G}\left(\left(\lambda\left(x^{-1} \circ y\right)_{-c+a}(x \circ y)_{c+a}\right)_{G, G}\left(x^{-1} \circ y\right)_{-c+d}(x \circ y)_{c+d}\right)_{G, G}
\end{array}\right) \\
& =\operatorname{rank}\left(e_{-a+b} e_{a+b}\right)_{G, G}
\end{aligned}
$$

with non-zero $\lambda$, where $\lambda$ depends on the homogeneous coordinates.

Proof. Replacing $x$ by $x^{-1}$ in (2), we know that the unique point $u=\left(u_{a}\right)_{G}$ in (2) is $x^{-1} \circ y$.

Proposition 1. 4 If $x \circ y$ is well-defined, then $y \circ x$ and $x \circ e(a)(a \in G)$ are also well-defined and they satisfy

(4) $x \circ y=y \circ x$,

(5) $x \circ e(a)=e(a) \circ x=x(a) \quad(a \in G)$,

(6) $e(a) \circ e(b)=e(a+b) \quad(a, b \in G)$.

This is an immediate consequence from the relation (3).

2. Since $\left(e_{-a+b} e_{a+b}\right)_{G, G}$ is symmetric, there exists a subset $H$ in $G$ such that the cardinal $|H|$ equals to the rank of $\left(e_{-a+b} e_{a+b}\right)_{G, G}$ and $\operatorname{det}\left(e_{-a^{\prime}+b}, e_{a^{\prime+b}}\right)_{H, H}$ $\neq 0$, where $\left(e_{\ldots a^{\prime}+b}, e_{a^{\prime}+b^{\prime}}\right)_{H, H}$ is an $|H| \times|H|$-matrix of which $\left(a^{\prime}, b^{\prime}\right)$-component is $e_{-a^{\prime}+b}, e_{a^{\prime}+b},\left(a^{\prime}, b^{\prime} \in H\right)$.

Using the inverse matrix

(7) $\left(\alpha_{a^{\prime}, b^{\prime}}\right)_{H, H}=\left(e_{-a^{\prime}+b}, e_{a^{\prime}+b}\right)_{H, H}^{-1}$,

we can express the relation (3) by the following explicite polynomial relations:

$$
\begin{aligned}
& \text { (8) } x_{-a+b} x_{a+b}=\sum_{c^{\prime}, d^{\prime} \in H} \alpha_{c^{\prime}, d^{\prime}} e_{-c^{\prime+a}} e_{c^{\prime+a}} x_{-d^{\prime+b}} x_{d^{\prime+b}} \\
& \text { (8') } y_{-a+b} y_{a+b}=\sum_{c^{\prime}, d^{\prime} \in H} \alpha_{c^{\prime}, d^{\prime}} e_{-c^{\prime}+a} e_{c^{\prime}+a} y_{-d^{\prime+b}} y_{d^{\prime+b}} \\
& \text { (8') } \lambda\left(x^{-1} \circ y\right)_{-a+b}(x \circ y)_{a+b}=\sum_{c^{\prime}, d^{\prime} \in H} \alpha_{c^{\prime}, d^{\prime}} x_{-c^{\prime}+a} x_{c^{\prime+a}} y_{-d^{\prime}+b} y_{d^{\prime}+b} \quad(a, b \in G)
\end{aligned}
$$

with non-zero $\lambda$. 
Definition 2. 1. We denote by $V_{e}$ the closed subscheme in $P_{G}$ which is the Zariski-closure of all the point $x$ such that $x^{-1} \circ x$ is well-defined and $x^{-1} \circ x=e$. We call $V_{e}$ the projective scheme associating with $e$.

Using $\left(\alpha_{a r, b}\right)_{H, H}$ we can define $V_{e}$ as the closed subscheme defined by the relations

(9) $\quad X_{-a+b} X_{a+b}=\sum_{c^{\prime}, a^{\prime} \in H} \alpha_{c^{\prime}, d^{\prime}} e_{-c^{\prime}+a} e_{c^{\prime}+a} X_{-d^{\prime}+b} X_{d^{\prime}+b}=0 \quad(a, b \in G)$

and

(10)

$$
\begin{aligned}
\sum \alpha_{c \prime, d}, & \left\{e_{c} X_{-c^{\prime}-a+b} X_{c^{\prime}-a+b} X_{-d^{\prime}+a+b} X_{d^{\prime}+a+b}\right. \\
& \left.-e_{a} X_{-c^{\prime}-c+b} X_{c^{\prime}-c+b} X_{-d^{\prime}+c+b} X_{d^{\prime}+c+b}\right\}=0 . \quad(a, b, c \in G) .
\end{aligned}
$$

Under what condition on $e=\left(e_{a}\right)_{G}$ the projective scheme $V_{e}$ is an abelian variety? This is very difficult problem, which is equivalent to giving the reasonable explicite generators of the relations between theta-constants. We shall be concerved with this problem in the next paper.

Institute of Mathematics Nagoya University. 